\title{
Multi institutional quantitative phantom study of yttrium-90 PET in PET/MRl: the MR-QUEST study
}

\author{
Nichole M. Maughan ${ }^{1}$, Mootaz Eldib ${ }^{2,3}$, David Faul ${ }^{4}$, Maurizio Conti ${ }^{5}$, Mattijs Elschot ${ }^{6}$, Karin Knešaurek \\ Francesca Leek ${ }^{8}$, David Townsend ${ }^{8}$, Frank P. DiFilippo ${ }^{9}$, Kimberly Jackson ${ }^{10}$, Stephan G. Nekolla ${ }^{11}$, \\ Mathias Lukas ${ }^{12,13}$, Michael Tapner ${ }^{14,15}$, Parag J. Parikh ${ }^{1 *}$ and Richard Laforest ${ }^{16}$
}

\author{
* Correspondence: \\ parikh@wustl.edu \\ 'Department of Radiation \\ Oncology, Washington University \\ School of Medicine, 4921 Parkview \\ Place, Campus Box 8224, St. Louis, \\ MO 63110, USA \\ Full list of author information is \\ available at the end of the article
}

\begin{abstract}
Background: Yttrium-90 $\left({ }^{90} \mathrm{Y}\right)$ radioembolization involves the intra-arterial delivery of radioactive microspheres to treat hepatic malignancies. Though this therapy involves careful pre-treatment planning and imaging, little is known about the precise location of the microspheres once they are administered. Recently, there has been growing interest post-radioembolization imaging using positron-emission tomography (PET) for quantitative dosimetry and identifying lesions that may benefit from additional salvage therapy. In this study, we aim to measure the inter-center variability of ${ }^{90} \mathrm{Y}$ PET measurements as measured on PET/MRI in preparation for a multi-institutional prospective phase I/II clinical trial.

Eight institutions participated in this study and followed a standardized phantom filling and imaging protocol. The NEMA NU2-2012 body phantom was filled with $3 \mathrm{GBq}$ of ${ }^{90} \mathrm{Y}$ chloride solution. The phantom was imaged for $30 \mathrm{~min}$ in listmode on a Siemens Biograph mMR non-TOF PET/MRI scanner at five time points across 10 days $(0.3-3.0 \mathrm{GBq})$. Raw PET data were sent to a central site for image reconstruction and data analysis. Images were reconstructed with optimal parameters determined from a previous study. Volumes of interest (VOls) matching the known sphere diameters were drawn on the vendor-provided attenuation map and propagated to the PET images. Recovery coefficients (RCs) and coefficient of variation of the RCs (COV) were calculated from these VOls for each sphere size and activity level.
\end{abstract}

Results: Mean RCs ranged from 14.5 to $75.4 \%$, with the lowest mean RC coming from the smallest sphere $(10 \mathrm{~mm})$ on the last day of imaging $(0.16 \mathrm{MBg} / \mathrm{ml})$ and the highest mean RC coming from the largest sphere $(37 \mathrm{~mm})$ on the first day of imaging $(2.16 \mathrm{MBq} / \mathrm{ml})$. The smaller spheres tended to exhibit higher COVs. In contrast, the larger spheres tended to exhibit lower COVs. COVs from the $37 \mathrm{~mm}$ sphere were $<25.3 \%$ in all scans. For scans with $\geq 0.60 \mathrm{MBq} / \mathrm{ml}, \mathrm{COV}$ s were $\leq 25 \%$ in spheres $\geq 22 \mathrm{~mm}$. However, for all other spheres sizes and activity levels, COVs were usually $>25 \%$.

Conclusions: Post-radioembolization dosimetry of lesions or other VOls $\geq 22 \mathrm{~mm}$ in diameter can be consistently obtained $(<25 \%$ variability) at a multi-institutional level using PET/MRI for any clinically significant activity for ${ }^{90} \mathrm{Y}$ radioembolization.

Keywords: Yttrium-90, PET, PET/MRI, Radioembolization 


\section{Background}

Yttrium-90 $\left({ }^{90} \mathrm{Y}\right)$ radioembolization involves the intra-arterial delivery of radioactive microspheres to primary or metastatic disease in the liver. ${ }^{90} \mathrm{Y}$ decays primarily with $\beta^{-}$emission (mean $0.937 \mathrm{MeV}, 64.2 \mathrm{~h}$ half-life, $2.5 \mathrm{~mm}$ mean soft tissue penetration, $11 \mathrm{~mm}$ max tissue penetration) [1], allowing for high amount of radiation dose within a well-confined region. The current commercially available microspheres, TheraSpheres (glass microspheres; BTG, London, UK) and SIR-Spheres (resin microspheres; Sirtex Medical, Sydney, Australia), were first approved in the USA by the Food and Drug Administration in 1999 and 2002 and received the European CE mark in 2005 and 2002, respectively [2, 3]. Since then, ${ }^{90} Y$ microsphere radioembolization has continued to grow as a third-line therapy for patients with some clinical trials into using this technology as a first-line therapy concurrent with FOLFOX-based chemotherapy [4]. While the use of ${ }^{90} \mathrm{Y}$ microspheres to treat cancer in the liver has been utilized for over 50 years [5], the treatment planning protocol from pre-treatment imaging to prescription activity calculation is not well optimized. Standard protocol does not allow for assessing the actual distribution of the microspheres or for predicting the effectiveness of treatment or possible toxicity events without waiting for follow-up imaging studies at least 30 days after treatment [6]. Patients who undergo ${ }^{90} \mathrm{Y}$ radioembolization typically have poor prognoses and earlier assessment of therapy immediately after injection could guide optimization of therapy.

Recently, there has been an interest in performing post-radioembolization imaging and dosimetry to assess the microsphere distribution and predict clinical outcomes. Early work involved Bremsstrahlung SPECT, utilizing the high Bremsstrahlung flux from the $\beta^{-}$emission of ${ }^{90} \mathrm{Y}$ [7-9]. However, these images suffer from poor spatial resolution, making it difficult to identify small areas of uptake and the heterogeneous distribution of the microspheres $[10,11]$ as well as perform quantitative evaluation. Early case studies found that the small positron yield $(0.0032 \%)$ of ${ }^{90} \mathrm{Y}$ was sufficient for imaging the microsphere distribution by PET [12-14]. Since then, several singlecenter studies have reported the ability to predict clinical outcomes using dosimetry derived from ${ }^{90} \mathrm{Y}$ PET images following radioembolization in both primary liver cancer [15] and metastatic colorectal cancer [16-18].

Multi-center clinical studies with quantitative end points, typically involves imaging a standard phantom to test quantitative accuracy and inter-center variability. Fahey et al. evaluated inter-center reproducibility and variability in a multi-center study involving fluorine-18 $\left({ }^{18} \mathrm{~F}\right)$ on PET/CT [19]. Another similar study was performed by Willowson et al. in the multi-center QUEST phantom study for measuring inter-site variability of ${ }^{90} \mathrm{Y}$ on PET/CT [20].

The purpose of this study is to measure both the inter- and intra-center variability of quantitatively measuring ${ }^{90} \mathrm{Y}$ on $\mathrm{PET} / \mathrm{MR}$ in preparation for a multi-center phase I/II clinical trial (NCT02611661). Our study mirrors the work that was performed by Willowson et al. in the QUEST study but, due to the difference in scanner designs and electronics, focuses instead on PET/MRI scanners. Furthermore, this study uses the same PET/MRI system model and identical reconstruction parameters across all centers, rather than allowing centers to reconstruction their own data with their own parameters. This was to test the true performance of the 
scanner with ${ }^{90} \mathrm{Y}$ without extra uncertainty introduced by varying choices in reconstruction parameters.

\section{Methods}

A total of eight institutions across four countries participated in this phantom study. All sites followed a strict protocol for both filling and imaging the phantom. Three NEMA NU2-2012 image quality (IQ) body phantoms (PTW Freiburg GmbH, Freiburg, Germany) with vendor-provided foam cradles and positioning devices were shared between the eight institutions.

\section{Phantom preparation}

Before filling the phantom with activity, the volume of the background compartment was measured by its water weight.

3.6 GBq in $1.4 \mathrm{~mL}$ of ${ }^{90} \mathrm{Y}$ chloride solution (PerkinElmer, Waltham, MA) was shipped to each institution. The supplier's calibrated activity listed on the shipping document was used as the "ground truth" for the activity within the vial. Each site recorded the amount of activity reported by their department's dose calibrator for comparison against the reported amount on the shipping label. The manufacturer and model of each site's dose calibrator is listed in Table 1.

The entire contents of the vial were completely emptied into $1300 \mathrm{~mL}$ of water with $100 \mathrm{mg}$ of either DTPA or EDTA added to prevent binding of ${ }^{90} \mathrm{Y}$ chloride to the walls of the phantom. Activity was drawn from this solution to fill the six spheres (diameters $37,28,22,17,13$, and $10 \mathrm{~mm}$ ). Once all spheres were filled, the remaining solution was emptied into the background compartment of the NEMA IQ phantom, and the remaining volume of the background compartment was filled with water. This resulted in an approximately 8:1 sphere-to-background activity concentration ratio. The center lung insert for the phantom was a solid structure made of polyethylene material. The total recorded activity in the phantom was the total activity listed on the shipping document minus any residual activity in the vial and syringe.

\section{Image acquisition and reconstruction}

The phantom was imaged at five time points (day 0 , day 3 , day 5 , day 7 , and day 10) at each institution on Siemens Biograph mMR non-time-of-flight (non-TOF)

Table 1 Dose calibrator and settings used at each site to measure ${ }^{90} \mathrm{Y}$ activity and verify against that reported on shipping label

\begin{tabular}{llll}
\hline Site number & Dose calibrator manufacturer & Model & Setting for ${ }^{90} \mathrm{Y}$ \\
\hline 1 & Capintec & CRC-12 & Channel $53 \times 10$ \\
2 & Capintec & CRC-55 T PET & Channel 010 \\
3 & Capintec & CRC-15R & Channel $48 \times 10$ \\
4 & Capintec & CRC-15R & Channel $56 \times 10$ \\
5 & Capintec & $55 \mathrm{t} \mathrm{W}$ & Channel $48 \times 10$ \\
6 & Veenstra Instruments & VIK 2025051 & Factor 902, scale 100 \\
7 & MED & ISOMED 2010 & Factor 2.3 (geometry: 3 ml syringe/1.4 ml solution) \\
8 & Capintec & CRC-15R & Channel 48 $\times 10$ \\
\hline
\end{tabular}


PET/MRI scanners, representing a range of total activities from 0.3 to $3.0 \mathrm{GBq}$. These values correspond to the full range of activities administered to patients treated with resin microspheres. The phantom was positioned in a foam cradle with an accompanying positioning device, as described by Ziegler et al. [21], to center the phantom in the field of view (FOV) and allow for reproducible placement of the phantom between scans and institutions. The phantom was imaged for $30 \mathrm{~min}$ in listmode acquisition in a single station. Due to the longer axial FOV of the Biograph mMR $(25.6 \mathrm{~cm})$, we are able to reduce the number of stations compared to other scanners with a shorter FOV (e.g., 15-16 cm), which typically require a minimum of two stations [20]. For post-radioembolization patients at our clinic (WUSM), we acquire PET data in a single station. At one of the sites, three back-to-back PET acquisitions (same phantom filling) were performed to evaluate intra-center variability. Another site (Washington University, St. Louis, MO) repeated the phantom filling with 54.4 MBq fluorine-18 $\left({ }^{18} \mathrm{~F}\right)$ and imaged at a single time point (15 min listmode acquisition) for comparison to a high statistic dataset. This ${ }^{18} \mathrm{~F}$ dataset was also reconstructed into ten smaller datasets, consisting of $3 \mathrm{~s}$ each of listmode data, to contain a similar amount of trues statistics to that seen in the ${ }^{90} \mathrm{Y}$ PET data. Those same ten data sets were reconstructed with additional randoms added to the sinograms to obtain a similar randoms fraction as that observed in the ${ }^{90} \mathrm{Y}$ PET data. A detailed description of these methods are described in [22]. This was to compare measurements from ${ }^{18} \mathrm{~F}$ data containing a similar amount of trues count statistics as that exhibited in ${ }^{90} \mathrm{Y}$ PET data on the highest activity day of imaging.

All raw PET data were sent via a secure data server (ABX-CRO Advanced Pharmaceutical Services, Dresden, Germany) to a central site (Washington University, St. Louis, MO) for reconstruction and analysis by a single investigator (N.M.). All PET reconstructions were performed using e7tools, the offline research reconstruction software provided by Siemens, with a vendor-provided, CT-derived attenuation map of the NEMA 2007/IEC 2008 Body phantom for attenuation correction, as described by Ziegler et al. [21] The attenuation map was manually inspected and registered for each PET data set since they were not measured directly from the PET/MRI scanner. Images were reconstructed with 3D ordinary Poisson ordered subset expectation maximization (OP-OSEM), with the following parameters determined from a previous phantom study: 3 iterations, 21 subsets, $5 \mathrm{~mm}$ full-width-half-maximum Gaussian postreconstruction filter, point spread function (PSF) compensation, and using absolute scatter correction with $4.17 \times 4.17 \times 2.03 \mathrm{~mm}^{3}$ voxels [23].

\section{Image post-processing and analysis}

All image post-processing, including drawing volumes of interest (VOIs) and extracting statistics from those VOIs, was performed in MIM v6.6.7 (MIM Software, Cleveland, $\mathrm{OH}$ ). VOIs were drawn on the attenuation map of the phantom (see Fig. 1). The VOIs on the spheres were drawn as spherical VOIs with a diameter matching the known sphere diameter. The VOIs with their corresponding purpose for quantitative assessment, according to NEMA NU 2-2007 guidelines, are summarized in Table 2. Each PET image was fused to the corresponding attenuation map for transfer of VOIs. 


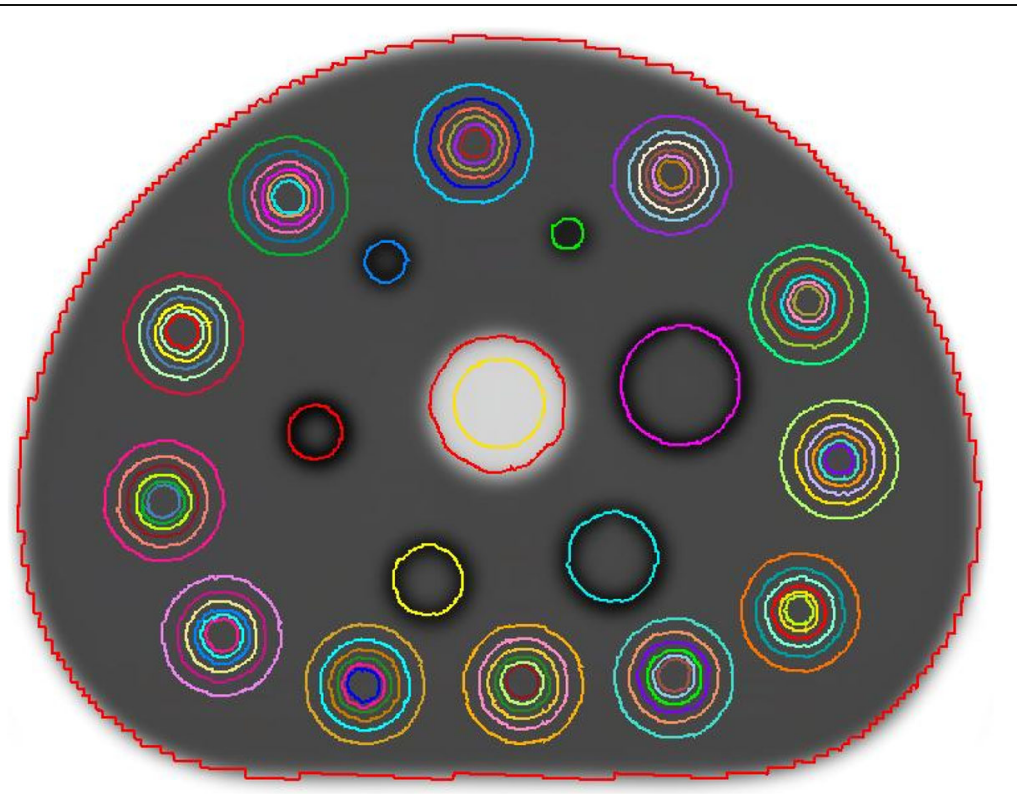

Fig. 1 Attenuation map of NEMA 2007/IEC 2008 body phantom with VOls drawn around each of the six fillable spheres, 72 VOls drawn in the background compartment, and a 28-mm diameter VOI drawn in the cold insert (center, yellow)

The recovery coefficients (RCs) for each of the fillable sphere and respective background VOIs were calculated to assess accuracy of measurements, especially in regards to partial volume effects (PVE):

$$
\mathrm{RC}(\%)=\frac{A_{m}}{A_{t}} \times 100
$$

where $A_{m}$ is the measured mean activity concentration and $A_{t}$ is the true activity concentration. The coefficient of variation (COV) of RCs for each hot sphere VOI and day of imaging ( 8 VOI measurements per COV) was used to quantify both inter- and intracenter variability:

$$
\operatorname{COV}_{i, n}(\%)=\frac{\sigma_{i, n}}{\mu_{i, n}} \times 100
$$

where $\sigma_{i, n}$ is the standard deviation and $\mu_{i, n}$ is the mean of RCs for a given sphere size (i) and day of imaging (n).

\begin{tabular}{|c|c|c|}
\hline VOI and size & Measured quantity & Purpose \\
\hline Field of view (FOV) & Total activity (GBq) & $\begin{array}{l}\text { Accuracy in total injected activity } \\
\text { measurement }\end{array}$ \\
\hline $\begin{array}{l}\text { Fillable spheres: } 37,28,22,17,13 \text {, } \\
10 \mathrm{~mm}\end{array}$ & $\begin{array}{l}\text { Recovery coefficient of mean } \\
\text { activity concentration }(\mathrm{MBq} / \mathrm{ml})\end{array}$ & $\begin{array}{l}\text { Accuracy in VOI measurements } \\
\text { from partial volume effects }\end{array}$ \\
\hline $\begin{array}{l}\text { Background spheres: } 12 \text { of } 37-\mathrm{mm} \\
\text { diameters }\end{array}$ & $\begin{array}{l}\text { Recovery coefficient of mean } \\
\text { activity concentration }(\mathrm{MBq} / \mathrm{ml})\end{array}$ & $\begin{array}{l}\text { Accuracy in warm background } \\
\text { VOI measurements }\end{array}$ \\
\hline $\begin{array}{l}\text { Background spheres: } 12 \text { each of 37-, 28-, } \\
\text { 22-, 17-, 13-, 10-mm diameters }\end{array}$ & $\begin{array}{l}\text { Standard deviation of mean } \\
\text { activity concentration }(\mathrm{MBq} / \mathrm{ml})\end{array}$ & $\begin{array}{l}\text { Variability in warm background } \\
\text { measurements (noise) }\end{array}$ \\
\hline Insert: 28-mm diameter, 160-mm length & $\begin{array}{l}\text { Mean activity concentration } \\
(\mathrm{MBq} / \mathrm{ml})\end{array}$ & Background counts \\
\hline
\end{tabular}

Table 2 Quantities for volume of interest (VOI) analysis 
Background variability for each sphere size $s\left(\mathrm{BV}_{\mathrm{s}}\right)$ for the highest activity day of imaging (12 measurements/site $\times 8$ sites $=96$ total VOIs) was calculated using

$$
\mathrm{BV}_{s}(\%)=\frac{\sigma_{s}}{\mu_{s}} \times 100
$$

where $\sigma_{s}$ is the standard deviation of the 96 background concentration measurements for a given sphere size $s$, and $\mu_{s}$ is the average of the 96 background concentration measurements for a given sphere size $s$.

Activity in the cold insert $\left(C_{\mathrm{i}}\right)$ was quantified using

$$
C_{i}(\%)=\frac{A_{m, i}}{A_{t, b}} \times 100
$$

where $A_{m, i}$ is the measured activity concentration in the cold insert, and $A_{t, b}$ is the true background activity concentration.

\section{Results}

Activities reported by each site's dose calibrator were on average $3.62 \%$ lower than that reported on the shipping label (median $-4.95 \%$, range $-6.31-0.61 \%$ ). Figure 2 shows transaxial PET images through the center of the spheres from the first day of imaging for each site.

After visual inspection and manual registration between the attenuation maps and the PET data, adequate registration was obtained in all data sets. Most data sets required approximately a few centimeters translation in the axial direction to properly align the attenuation map with the PET data. At some sites, the phantom was positioned in a reversed direction or the center plate containing the spheres was rotated (Fig. 2), in which case a rotation was required to properly align the attenuation map with the data.

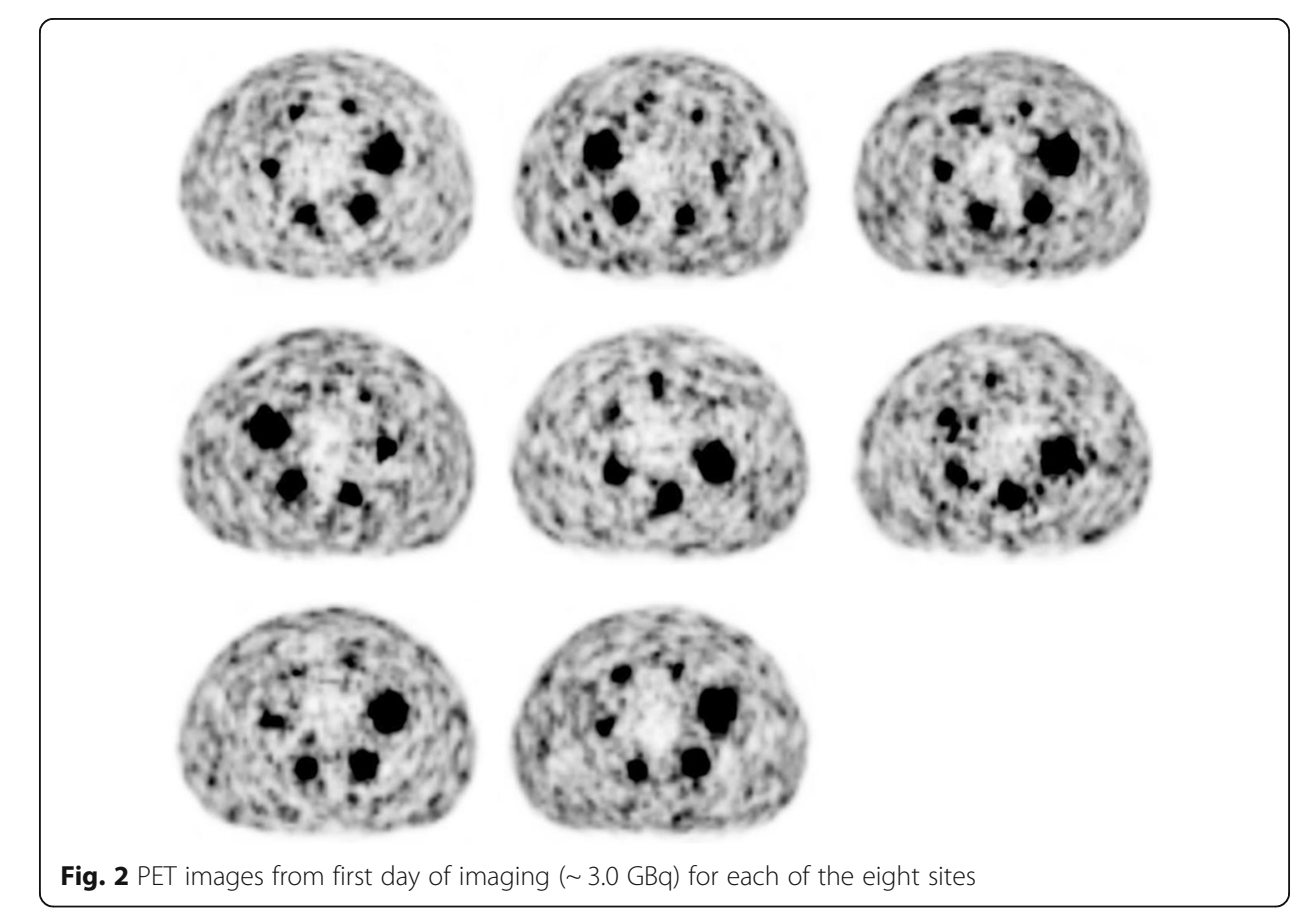


Figure 3a shows the measured activity versus true activity within the whole FOV along the identity line, averaged across the eight sites, and Fig. 3b shows the percent differences in total measured activity and true activity. The total activity measured within the FOV had a median error of $-0.63 \%$ across all activity ranges from all sites ( 8 sites $\times 5$ days of imaging $=40$ measurements) $($ mean $4.71 \%$, range $-23.9-65.0 \%)$. All average measurements of total activity were within $\pm 10 \%$ of true activity for activities $\geq$ $0.5 \mathrm{GBq}$. The lowest activity measurements $(\sim 0.3 \mathrm{GBq})$ overestimated the total activity within the FOV on average by $25.8 \%$ (range $-20.8-65.0 \%$ ).

Figure $3 \mathrm{c}$ shows the mean measured versus true activity concentration in the $37 \mathrm{~mm}$ VOI in the warm background compartment of the phantom along the identity line, and Fig. 3d shows the percent deviation in this background activity concentration. Mean measured activity concentration had a median error of $-1.68 \%$ across all activity ranges from all sites ( 12 measurements/site $\times 5$ imaging days $\times 8$ sites $=480$ measurements $)$ (mean $-1.43 \%$, range $-31.3-32.1 \%$ ).

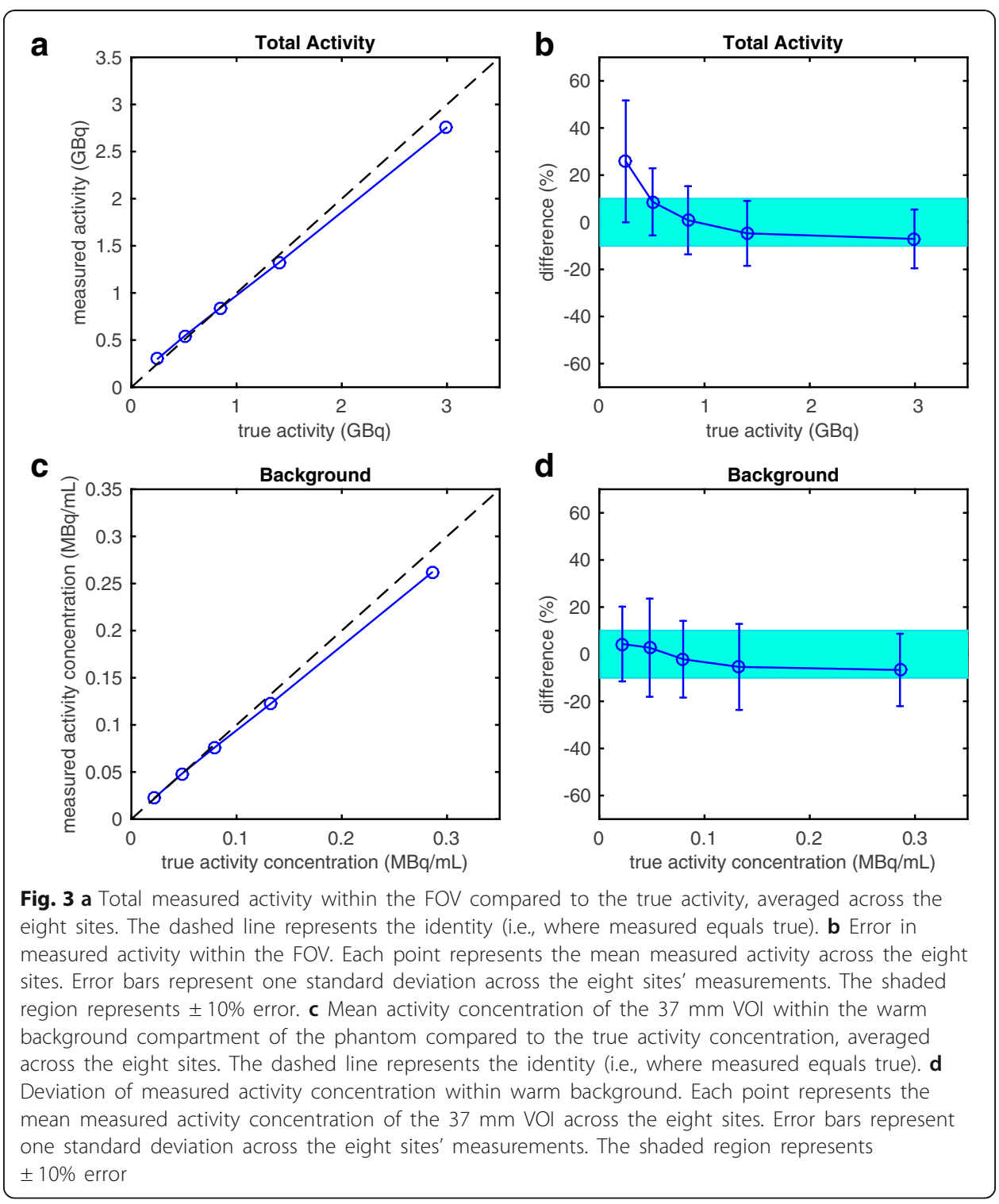


Figure 4 shows the noise in the PET imaging volumes, quantified by both the background variability (Eq. 3, Fig. 4a) and the signal intensity in the cold lung insert normalized by the true background activity (Eq. 4, Fig. 4b). The median background variability ranged from 6.48 to $32.2 \%$, measured from the largest and the smallest spheres, respectively, across all sphere sizes (total of 576 warm background VOIs), with a median variability of $16.5 \%$ (mean $16.7 \%$ ). The median cold insert counts $\left(C_{i}\right)$ increased from 33.3 to $75.6 \%$ in the lung insert from $0.29 \mathrm{MBq} / \mathrm{ml}$ to the lower true activity concentrations of $0.02 \mathrm{MBq} / \mathrm{ml}$. The median cold insert counts percentage across 40 PET imaging volumes was $45.3 \%$ of the true warm background activity concentration (mean 48.8\%, range 23.9-104\%). In comparison, the cold insert counts normalized to warm background in the ${ }^{18} \mathrm{~F}$ phantom image was $26 \%$.

Figure 5 and Table 3 illustrate the RCs and variation of each sphere size averaged over the different institutions. The inter-center variability of imaging with ${ }^{90} \mathrm{Y}$, expressed as the standard deviation on the RC measured at the different sites, is shown on the graphs as the error bars. A comparative measurement done with the same phantom but with high statistics ${ }^{18} \mathrm{~F}$ is indicated as the solid line on the graphs. The median $\mathrm{RC}$ for each hot sphere across all sites and activity concentrations (5 imaging days $\times 8$ sites $=40$ measurements per sphere) was $68.7,52.4,46.8,41.8,27.5$, and $22.0 \%$ for the 37-, 28-, 22-, 17-, 13-, and 10-mm spheres, respectively. The highest mean RC for any given activity concentration and sphere size was $75.4 \%$, measured on the $37-\mathrm{mm}$ sphere at the highest activity $(\sim 2.16 \mathrm{MBq} / \mathrm{ml})$. In contrast, the lowest mean $\mathrm{RC}$ for any given activity concentration and sphere size was $14.5 \%$, measured from the $10-\mathrm{mm}$ sphere at the lowest activity level $(\sim 0.16 \mathrm{MBq} / \mathrm{ml})$. Agreement with ${ }^{18} \mathrm{~F}$ measurements was best for the largest sphere and at the two highest activity levels $(1.00$ and $2.16 \mathrm{MBq} / \mathrm{ml}$ of $\left.{ }^{90} \mathrm{Y}\right)$, with deviation of -1.7 and $-1.5 \%$, respectively. Variability in measurements increased with decreasing sphere size and decreasing activity.

Figure $6 \mathrm{a}-\mathrm{e}$ and Table 4 illustrate the $\mathrm{RC}$ variability obtained from one site (Norwegian University of Science and Technology, Trondheim, Norway) who acquired three consecutive scans for each imaging day. The median RC for each hot sphere across the three scans and all activity concentrations ( 5 imaging days $\times 3$ scans $=15$ measurements per sphere) was $74.8,56.9,55.6,47.2,44.1$, and $24.3 \%$ for the 37-, 28-, 22-, 17-, 13-, and 10-mm spheres, respectively. . At the highest activity level, variability between scans for all hot
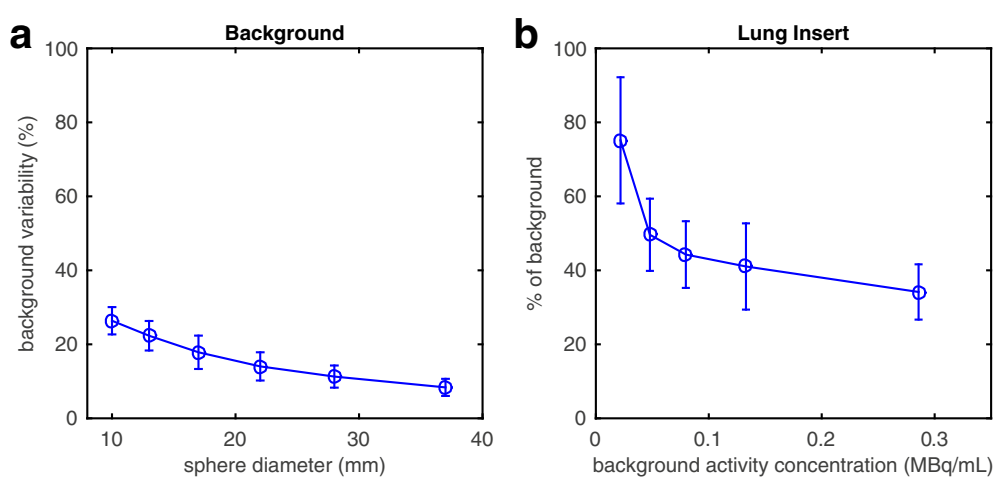

Fig. 4 a Variability in measurements between $12 \times 8$ background VOls for each sphere size. b Misplaced counts in cold lung insert reported as percent of true background activity concentration. Each point represents the mean. Error bars represent one standard deviation 


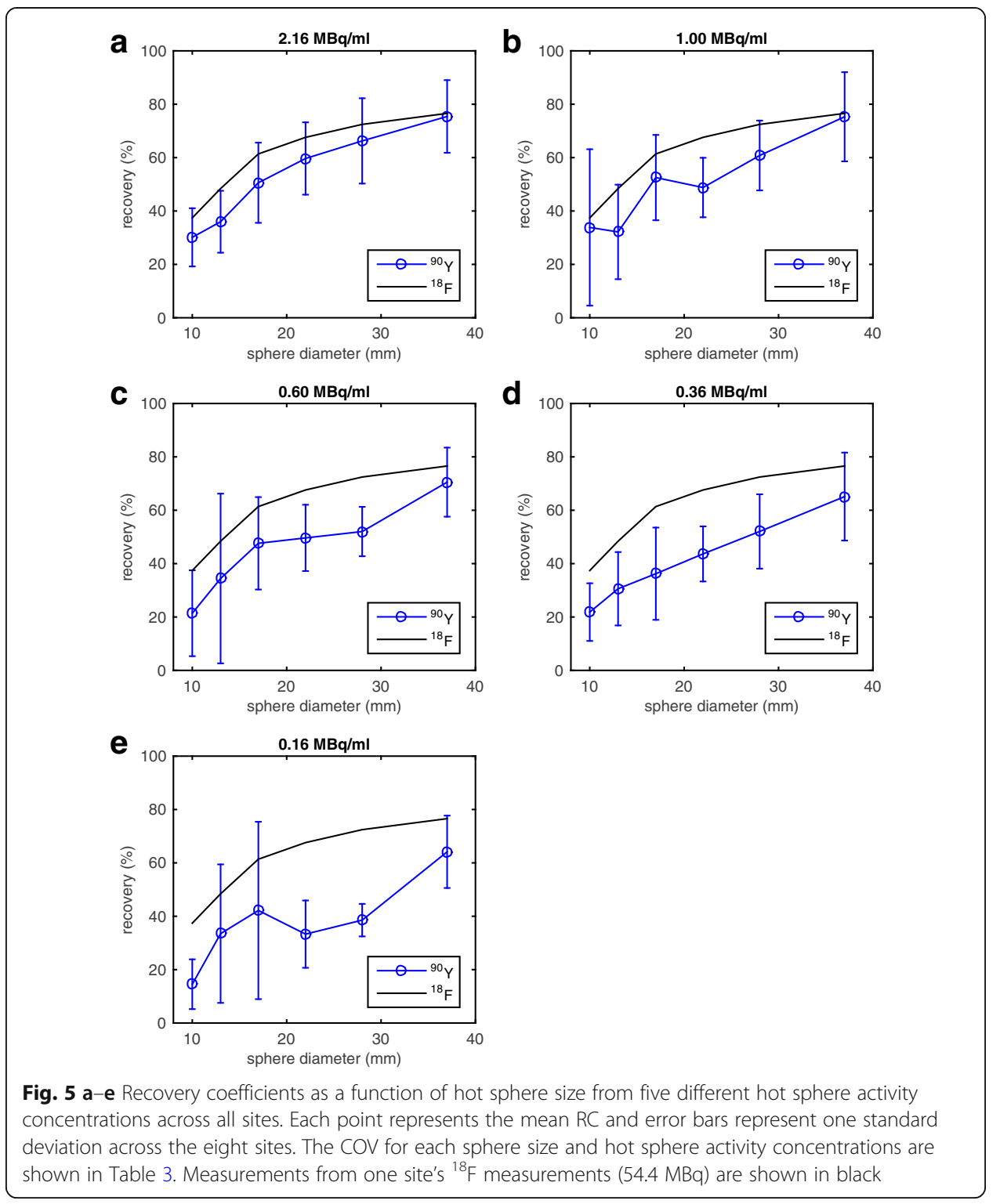

Table 3 Inter-center coefficient of variation (COV) of recovery coefficients (RCs) for each sphere size and activity concentration

\begin{tabular}{llllll}
\hline \multirow{2}{*}{ Sphere diameter $(\mathrm{mm})$} & \multicolumn{6}{l}{ COV $(\%)(\mathrm{range})$} \\
\cline { 2 - 6 } & $2.16 \mathrm{MBq} / \mathrm{ml}$ & $1.00 \mathrm{MBq} / \mathrm{ml}$ & $0.60 \mathrm{MBq} / \mathrm{ml}$ & $0.36 \mathrm{MBq} / \mathrm{ml}$ & $0.16 \mathrm{MBq} / \mathrm{ml}$ \\
\hline 10 & $36.2(14.0-48.6)$ & $86.6(6.18-99.8)$ & $75.1(7.79-51.7)$ & $49.5(9.83-45.1)$ & $64.1(2.63-26.9)$ \\
13 & $32.2(23.5-59.8)$ & $55.1(18.0-68.3)$ & $92.3(12.5-112)$ & $44.9(13.6-49.6)$ & $77.5(3.47-65.5)$ \\
17 & $29.7(18.9-69.1)$ & $30.4(30.3-78.0)$ & $36.4(27.5-75.8)$ & $47.7(20.5-75.0)$ & $78.8(14.7-118)$ \\
22 & $22.7(41.2-84.1)$ & $22.8(29.6-63.4)$ & $25.0(32.6-70.0)$ & $23.7(30.5-56.1)$ & $37.9(10.4-49.4)$ \\
28 & $24.1(42.9-88.1)$ & $21.5(41.7-80.3)$ & $17.8(38.0-65.3)$ & $26.8(26.8-69.6)$ & $15.8(26.8-46.9)$ \\
37 & $18.0(56.0-95.0)$ & $22.2(52.0-103)$ & $18.3(54.9-92.2)$ & $25.3(46.4-91.4)$ & $21.1(45.2-81.3)$ \\
\hline
\end{tabular}

Values in parentheses represent the range of RCs (min-max) 

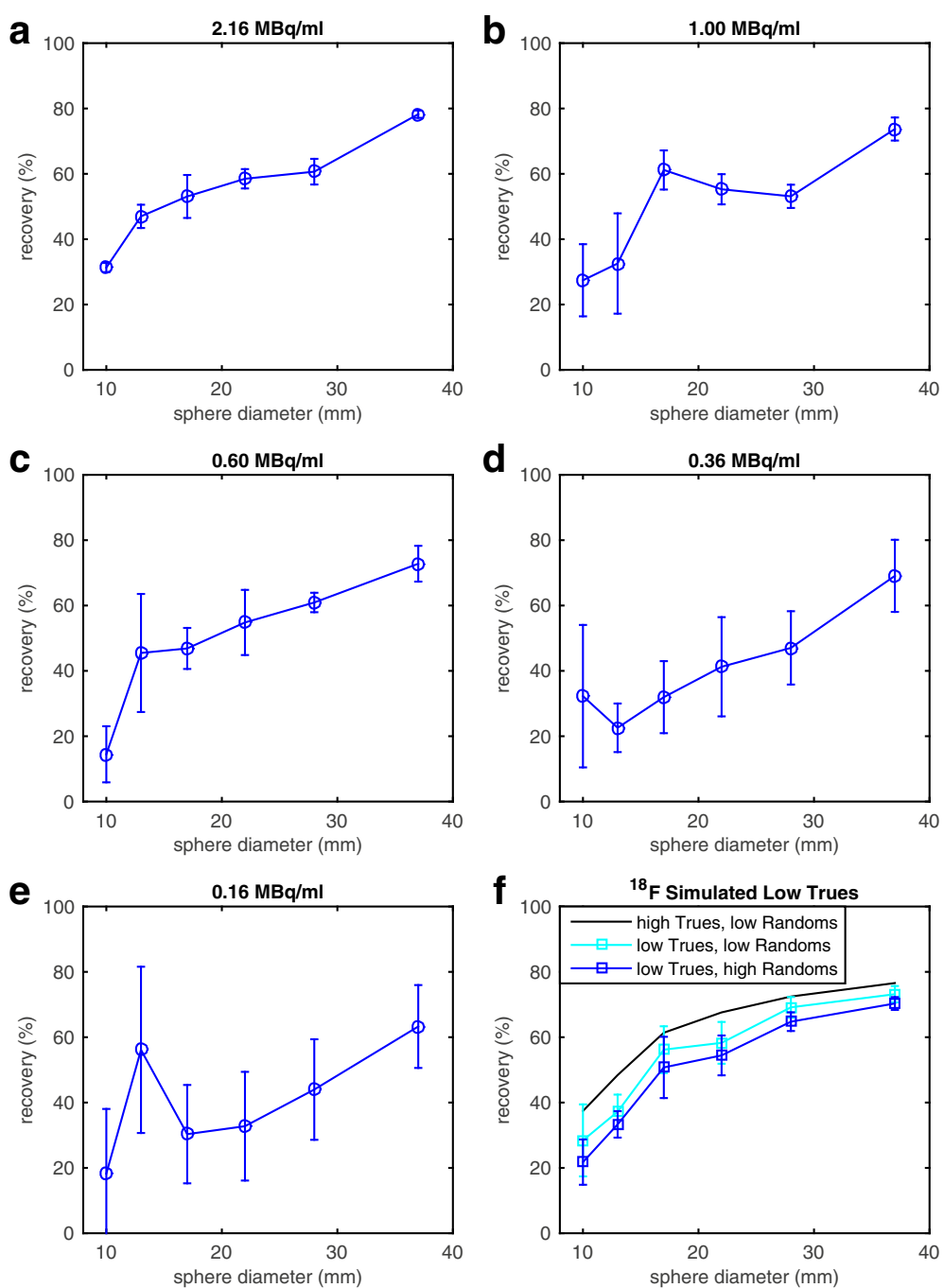

Fig. 6 a-e Recovery coefficients as a function of hot sphere size from three consecutive scans at a single site. Each point represents the mean RC, and error bars represent one standard deviation. The COV for each sphere size and hot sphere activity concentrations are shown in Table 4. $\mathbf{f}$ Measurements from raw ${ }^{18} \mathrm{~F}$ data (black line), ${ }^{18} \mathrm{~F}$ data with similar ${ }^{90} \mathrm{Y}$ true count statistics (light blue square), and ${ }^{18} \mathrm{~F}$ data with similar true count statistics and randoms fraction to ${ }^{90} \mathrm{Y}$. Again, each point represents the mean RC and error bars represent one standard deviation across the ten $3-\mathrm{s}^{18} \mathrm{~F}$ datasets

Table 4 Intra-center coefficient of variation (COV) of recovery coefficients (RCs) for each sphere size and activity concentration

\begin{tabular}{llllll}
\hline \multirow{2}{*}{ Sphere diameter $(\mathrm{mm})$} & \multicolumn{1}{l}{ COV (\%) (range) } & & & & \\
\cline { 2 - 6 } & $2.32 \mathrm{MBq} / \mathrm{ml}$ & $1.06 \mathrm{MBq} / \mathrm{ml}$ & $0.65 \mathrm{MBq} / \mathrm{ml}$ & $0.38 \mathrm{MBq} / \mathrm{ml}$ & $0.17 \mathrm{MBq} / \mathrm{ml}$ \\
\hline 10 & $4.49(29.7-32.3)$ & $40.2(20.8-40.2)$ & $59.4(8.71-24.3)$ & $67.6(14.4-56.6)$ & $108(4.42-40.9)$ \\
13 & $7.58(44.1-51.0)$ & $47.1(22.6-50.2)$ & $39.7(27.2-63.3)$ & $32.9(16.6-30.9)$ & $45.3(29.1-79.6)$ \\
17 & $12.4(48.8-60.6)$ & $9.79(57.5-68.1)$ & $13.4(40.4-53.0)$ & $34.5(21.6-43.5)$ & $49.6(15.6-45.7)$ \\
22 & $5.06(55.6-61.5)$ & $8.35(50.2-59.2)$ & $18.2(43.4-62.0)$ & $36.8(27.8-57.7)$ & $50.8(14.5-47.1)$ \\
28 & $6.48(56.2-63.7)$ & $6.69(49.9-57.0)$ & $4.89(58.9-64.3)$ & $23.9(34.9-56.9)$ & $35.0(27.2-57.4)$ \\
37 & $1.43(77.0-79.0)$ & $4.79(70.1-77.1)$ & $7.52(66.6-77.0)$ & $16.0(56.6-77.4)$ & $20.0(53.9-77.7)$ \\
\hline
\end{tabular}

Values in parentheses represent the range of RCs (min-max) 
spheres was $<13 \%$ (mean $6.24 \%$, median $5.77 \%$, range $1.42-12.4 \%$ ). All spheres $\geq 17 \mathrm{~mm}$ with $\geq 0.60 \mathrm{MBq} / \mathrm{ml}$ had $<20 \%$ variability in their RCs. The $37-\mathrm{mm}$ sphere had a variability in measurements $\leq 20 \%$ for all activity levels. Again, variability increased with decreasing sphere size and decreasing activity. Table 5 lists the COV and range for these scans for $\mathrm{RC}$ of total activity and background activity concentration and $C_{i}$ values of the cold lung insert. Variability tended to increase with decreasing activity for these VOIs as well, with the exception of total activity, which tended to remain rather stable. Figure $6 \mathrm{f}$ shows measurements from the ${ }^{18} \mathrm{~F}$ phantom, reconstructed into smaller data sets with both trues counts statistics and/or randoms fraction typically seen with ${ }^{90} \mathrm{Y}$ PET data from the highest activity imaging day. Total trues for these smaller ${ }^{18} \mathrm{~F}$ data sets were $2.07 \times 10^{6}$ and those reported by one site for ${ }^{90} \mathrm{Y}$ were $1.54 \times 10^{6}$. The randoms fraction for typical ${ }^{18} \mathrm{~F}$ datasets was $31 \%$ and that for the simulated high-randoms was $77 \%$. The randoms fraction for ${ }^{90} \mathrm{Y}$ was approximately $75 \%$.

\section{Discussion}

Despite the low positron yield from ${ }^{90} \mathrm{Y}$, PET imaging of this isotope has proven both feasible and useful in previous literature [11-13, 15-17, 23, 24] and is further demonstrated in this work. The results from this study and that reported by Willowson et al. in the PET/CT QUEST study [20] also demonstrate the feasibility of performing multiinstitutional clinical studies focused on ${ }^{90} \mathrm{Y}$ PET-based dosimetry.

Even though this multi-institutional phantom study with ${ }^{90} \mathrm{Y}$ PET imaging has been performed previously on PET/CT [20], there are several key differences between Siemens Biograph PET/CT scanners and Siemens Biograph mMR (PET/MRI) scanners that motivate the need to perform this study on PET/MRI. First, the Biograph mMR utilizes avalanche photodiode (APD) detectors instead of typically used photomultiplier tubes (PMT), to allow for compatibility with the MR magnetic field. However, this comes at the cost of not having TOF capabilities. Additionally, compared to the Biograph PET/CT scanners, the mMR exhibits increased sensitivity due to the geometrical arrangement of the detectors: $15.0 \mathrm{kcps} / \mathrm{MBq}$ ) [25] versus $8.1 \mathrm{kcps} / \mathrm{MBq}$ on the Biograph 40 (non-TOF) PET/CT [26]. As discussed previously, the difference in sensitivities is due to a longer axial FOV and shorter ring diameter on the Biograph mMR [23]. The tight geometry and wider coincidence timing window in mMR result in higher random rates, but the smaller block size results in lower singles rate per detector block in comparison to Siemens PET/CT scanners [23]. All of these factors (i.e., difference in electronics and higher sensitivity from a different geometry), combined with low annihilation counts and high randoms rates from Bremsstrahlung radiation, make

Table 5 Intra-center coefficient of variation (COV) between three consecutive scans of recovery coefficients (RCs) of total activity and background activity concentration and of counts in the cold lung insert $\left(C_{i}\right)$ on each imaging day

\begin{tabular}{|c|c|c|c|c|c|}
\hline \multirow[b]{2}{*}{ VOI } & \multicolumn{5}{|l|}{ COV (\%) (range) } \\
\hline & $3.0 \mathrm{GBq}$ & 1.4 GBq & $0.8 \mathrm{GBq}$ & $0.5 \mathrm{GBq}$ & $0.2 \mathrm{GBq}$ \\
\hline Total activity & $0.80(96.7-98.2)$ & $0.47(102-102)$ & $0.78(106-107)$ & $0.79(114-115)$ & $0.80(139-141)$ \\
\hline Background & $16.8(46.5-147)$ & $26.9(33.6-209)$ & $27.9(32.8-179)$ & $34.6(32.8-254)$ & $50.4(30.5-423)$ \\
\hline Lung insert & $4.51(31.5-34.5)$ & $5.99(39.6-44.6)$ & $2.82(37.4-39.1)$ & $5.05(39.9-44.5)$ & $10.2(61.1-74.1)$ \\
\hline
\end{tabular}

Numbers in parentheses represent the range of RCs for total activity and background activity concentration and $C_{i}$ for the lung insert (min-max) 
the similarity of the convergence properties of OSEM imaging reconstruction different between Biograph PET/CT and Biograph PET/MRI scanners. This was demonstrated in our previous phantom study, where we found that the optimal reconstruction parameters, using the same number of subsets, post-reconstruction filter size, and resolution recovery, were at three iterations on the mMR instead of two iterations for Biograph PET/CT scanners, as suggested by Willowson et al. [20]. Therefore, it is necessary to demonstrate the quantitative accuracy and test the variability between scanners on mMR, especially since this has not been done.

${ }^{90} \mathrm{Y}$ has high randoms rates (due to a high flux of Bremsstrahlung radiation) and low trues statistics (due to low annihilation counts); therefore, a careful handling of the randoms in the reconstruction proves essential in iterative reconstruction algorithms. In previous generations of OSEM, the pre-subtraction of randoms and scatter results in significant bias [27, 28]. Siemens mMR uses 3D OP-OSEM with PROMPTS+RANDOMS data acquisition, as done in Biograph $\mathrm{mMR}$ and $\mathrm{mCT}$, there is no pre-subtraction of scatter or randoms estimates, and therefore the algorithm bias is reduced [29]. However, in situations of very low counts such with ${ }^{90} \mathrm{Y}$ imaging, the convergence properties of the algorithm may be such that more iterations are needed at the expense of increased noise. In our phantom evaluation [23], we limited the noise in the images by stopping after three 3D OP-OSEM iterations (with 21 subsets resulting in 63 updates) and applying a $5 \mathrm{~mm}$ Gaussian post-reconstruction filter. As shown in [23], images reconstructed with more iterations and sharper filter resulted in unacceptably noisy images. The low count statistics and high randoms fraction for ${ }^{90} \mathrm{Y}$ PET imaging are likely the cause of lower count recovery compared to higher statistics ${ }^{18} \mathrm{~F}$ PET imaging. Evidence of this is shown in Fig. 6f. Optimized image reconstruction algorithms, with extremely low count statistics and high random counts, have yet to be resolved [20, 23, 30]. This is an area of future research.

Recovery of the total activity within the whole FOV was consistent among sites, with most measurements within $\pm 10 \%$ of the true values, especially above $0.5 \mathrm{GBq}$. These values are consistent with those reported by Willowson et al. in the PET/CT QUEST study for 19 Siemens Biograph TOF PET/CT scanners [20], though our reported standard deviation on the Biograph mMR at $0.5 \mathrm{GBq}$ was slightly higher, likely due to a smaller number of sites. Our total activity results were closer in agreement with the expected activity than those reported for Siemens Biograph non-TOF PET/CT scanners in the same QUEST study, where mean error in the FOV at $0.5 \mathrm{GBq}$ was approximately $+20 \%$ and standard deviation in this error was approximately $\pm 60 \%$ [20]. Our study performed an extra scan at a lower activity level $(0.3 \mathrm{GBq})$ that was not performed in the PET/CT QUEST study. The extra scan at a lower activity level represents the subset of patients who are administered the lowest activity available for resin microsphere treatment. Total activity measurements in this range were, on average, overestimated by $>20 \%$, with a standard deviation reaching beyond $\pm 20 \%$. This trend in overestimation of total activity with decreasing activity has been reported in previous studies using both TOF and non-TOF Siemens Biograph PET/CT scanners [20, 24]. A possible explanation could be extremely low count statistics resulting in higher noise, uncertainty in scatter correction, or artificial peaks in the data resulting in measured activity higher than the true activity. This effect may be less prominent in TOF reconstructions, 
since it is known that TOF reduces noise by reducing noise propagation in both forward- and back-projections at each iteration [31].

Activity measurements in the warm background compartment of the phantom were excellent, with the mean error consistently $<10 \%$. These values are consistent with those reported in the QUEST study for both the Siemens Biograph TOF ( $>1$ iteration, no post-reconstruction filter reconstructions only) and non-TOF PET/CT (PROMPTS+RANDOMS reconstructions only) scanners [20]. The standard deviations in our warm background measurements were also comparable to those reported in the QUEST PET/CT study. It is important to note that the background compartment of the phantom was intended to simulate the non-zero uptake of normal liver parenchyma, as is commonly seen in clinical studies [15, 16]. Even though liver tumors tend to take up higher concentrations of the ${ }^{90} \mathrm{Y}$ microspheres, which is the goal of radioembolization, the normal liver parenchyma often takes up some of the microspheres as well, albeit usually at a lower concentration. Thus, it was imperative to include warm background activity in the large compartment of the phantom in an attempt to simulate the clinical setting. In our experience, the ratio of tumor:normal liver ("background") activity concentrations varies from patient to patient. Future work includes investigating the effects of tumor:background activity concentrations on image quality.

Noise in the PET imaging volumes was quantified by both the background variability on the high activity day of imaging $(\sim 0.29 \mathrm{MBq} / \mathrm{ml})$ (Eq. 3) and the scatter/background counts in the cold (no activity) lung insert of the phantom (Eq. 4). Background variability on the Biograph mMR was significantly lower than that reported for both the TOF and non-TOF Biograph PET/CT scanners in the QUEST study $(16.7 \%$ mean versus 30, 50, and 38\% means for TOF and Gaussian post-reconstruction filter, TOF and no post-reconstruction filter, and non-TOF reconstructions, respectively) [20]. However, scatter counts in the lung insert from the mMR (49.0\% mean) were higher than those from Biograph TOF PET/CT scanners ( 30\% mean) and non-TOF PET/CT scanners using PROMPTS+RANDOMS mode ( 35\% mean). They were, however, lower than those from Biograph non-TOF PET/CT scanners in NETTRUES mode $(\sim 60 \%$ mean $)$ [20]. A possible reason for the higher rate of scatter counts in the cold lung insert compared to those reported by Willowson et al. could be due to fact that the attenuation maps from this study were vendor-provided, since attenuation maps of phantoms cannot be directly measured with MRI. Depending on the type of lung insert used in the vendor provided maps (styrofoam or solid), this could affect the attenuation properties and scatter estimates used in the reconstruction. However, private communication with a representative from Siemens confirmed that the vendor-provided attenuation map was measured from identical phantoms used in this study. Another possibility is that the difference in geometry of the Biograph mMR scanner (i.e., longer axial FOV) may allow for more scatter counts than the Biograph mCT or non-TOF PET/CT scanners. Regardless, scatter counts are higher for any of these three scanners compared to ${ }^{18} \mathrm{~F}$ when imaging ${ }^{90} \mathrm{Y}$. A possible explanation is that in very low count studies, the estimated scatter distribution may be inaccurate. The scatter distribution is iteratively estimated from the measured activity distribution and the phantom attenuation map. When count statistics are low in an imaging dataset, residual scatter counts may remain in regions of cold (i.e., no) activity, and the data supports this theory. For the 
low activity scan, the counts in the cold insert were approximately $76 \%$ of warm background, which is significantly higher than that measured for ${ }^{18} \mathrm{~F}$ at $26 \%$. At the highest ${ }^{90} \mathrm{Y}$ activity scan, cold insert counts was approximately $33 \%$ of warm background, much closer to that of ${ }^{18} \mathrm{~F}$. Higher residual scatter counts in cold areas may also explain why hot sphere RCs for low total activity scans are much lower that for ${ }^{18} \mathrm{~F}$. Others have investigated incomplete convergence in these cold areas [32], and this remains an area of active research.

Count recovery in the higher activity concentrated (hot) spheres was good, with mean RCs ranging from approximately $30-75 \%$ on the highest activity imaging day $(\sim 2.16 \mathrm{MBq} / \mathrm{ml})$. These values are consistent with those from a previous ACR phantom study at two of the institutions included in this study [23]. They are also consistent with those reported for the same total activity level in the QUEST study for the Siemens Biograph TOF PET/CT scanners (two iterations, 5-mm Gaussian post-reconstruction filter) and better than those for the non-TOF PET/CT scanners (all reconstructions) [20]. RCs from ${ }^{90} \mathrm{Y}$ PET imaging were also lower than those from ${ }^{18} \mathrm{~F}$ PET imaging, with the exception of the largest sphere size at the highest activity. We report lower ${ }^{18} \mathrm{~F}$ RCs than Willowson et al., who reported RCs approaching near 100\% for the largest sphere [20]. Discrepancy in these measurements is likely due to a difference in the method for drawing VOIs: they used a region-growing approach, where VOIs were drawn at 50\% of the maximum value, whereas we used the known sphere diameter to draw VOIs. Using an attenuation map-based method for drawing VOIs, as opposed to a region-growing approach, is known to decrease RCs in PET images [24]. Our method is more susceptible to partial volume effects but is more representative of what is performed for individual lesion dosimetry. As is characteristic of PET imaging studies, whether using ${ }^{90} \mathrm{Y}$ or a standard isotope-like ${ }^{18} \mathrm{~F}$, RCs degraded with decreasing VOI size [21, 23, 24, 33, 34]. Combining this with the high noise, which is characteristic of ${ }^{90} \mathrm{Y}$ PET images, it is apparent from Fig. 2 that very small regions of high uptake (i.e., the smallest sphere) are difficult, if not impossible, to identify. This is a limitation that must be considered in clinical applications of ${ }^{90} \mathrm{Y}$ PET imaging when assessing microsphere uptake in small lesions. RCs of any given hot sphere size also slightly decreased with decreasing activity concentration, similar to previous phantom studies [20, 23]. Contributing factors to sub-optimal recovery, especially below ${ }^{18} \mathrm{~F}$, could include partial volume effects, low positron statistics, and high randoms rates. Low annihilation counts, and thus low trues rates, especially combined with high randoms rates, are limiting factors of OSEM reconstruction algorithms, as previously discussed. Low trues rates are demonstrated as a contributing factor for poorer recovery in Fig. $6 \mathrm{f}$ for even a standard isotope such as ${ }^{18} \mathrm{~F}$. However, this does not account for the entire discrepancy between these two isotopes. Handling these combined factors in iterative reconstruction algorithms is an active area of research, not just in ${ }^{90} \mathrm{Y}$ PET imaging [30] but also in gated-cardiac PET imaging where statistics are often low [35].

Inter-center variability, as quantified by the COV for each sphere size and activity concentration, was acceptable $(<25 \%)$ for sphere diameters $\geq 22 \mathrm{~mm}$ and $\geq 0.60 \mathrm{MBq} / \mathrm{ml}$. For the highest activity level, inter-center variability was $<23 \%$ for sphere diameters $\geq 22 \mathrm{~mm}$. In the multi-institutional phantom study by Fahey et al., nine sites tested the variability of imaging ${ }^{18} \mathrm{~F}$ on PET/CT using an ACR phantom in preparation for a multi-institutional clinical trial. They reported COVs in RCs of 5.9, 21.2, and 17.0\% for VOI diameters 25, 16, 
and $12 \mathrm{~mm}$, respectively [19]. Though these values are lower than what we measured for our comparably sized sphere VOIs, considering the noisy nature of ${ }^{90} \mathrm{Y}$ PET images, our results offer promise for the ability to reliably perform multi-institutional clinical studies of ${ }^{90} \mathrm{Y}$ PET-based dosimetry with the Siemens Biograph mMR.

Intra-center variability was also acceptable, $<7 \%$ for sphere diameters $\geq 22 \mathrm{~mm}$, which was lower than that reported by the QUEST study for the Biograph TOF PET/CT (5, 4, and $8 \%$ for sphere diameters 37,28 , and $22 \mathrm{~mm}$, respectively) on the highest activity imaging day $(2.32 \mathrm{MBq} / \mathrm{ml})$ [20]. Yet, for low activity concentrations, this variability increased significantly, and COVs of the spheres became comparable to those seen at a multi-institutional level, suggesting that inter-center variation at low activities is likely more due to scanner performance than experimental error. Conversely, the large variability seen at the inter-center level at even the highest activity concentration (Fig. $5 \mathrm{a}-\mathrm{c}$ ) that is generally not present at the intra-center level for these same activity concentration ranges (Fig. 6a-c) suggests there could be discrepancies in the phantom preparation in addition to differences between individual scanner performance.

Several limitations exist with this study. As mentioned previously, we used a vendor-provided attenuation map since direct attenuation map acquisition of phantoms is not accurate on PET/MRI scanners. A study by Ziegler et al. found that the MR-derived attenuation map of the NEMA image quality phantom, obtained from the Siemens Biograph mMR PET/MRI scanner, only captured the photon attenuation of water inside the phantom but failed to capture the plastic housing of the phantom itself, which significantly degraded both the quantitative accuracy and background variability of the PET images. Image quality was improved when a CT-derived attenuation map of the phantom was used instead [21]. It is important to note that this necessity for using a CT-derived attenuation map only applies to phantom studies and is not necessary for clinical studies. Since the attenuation maps were not measured directly from the PET/MRI scanner at the time of PET imaging, they had to be manually registered and verified to the PET volumes in order to incorporate into offline reconstruction. Manual registration of the attenuation map to the PET data may have introduced error during the reconstruction procedure since it is not an exact process; however, visual inspection confirmed satisfactory registration between the data sets. We attempted to further mitigate any re-positioning errors both at the intra- and inter-center level by using a phantom cradle and positioning device to replicate phantom placement in the scanner between sites. Furthermore, since we were unable to image the phantom's plastic housing directly with MRI, we could not see if the spheres were filled completely; thus, the "true" activities may have actually been overestimates of what was actually filled in the spheres. Since our RCs agreed well with those reported in the QUEST study by Willowson et al. for Siemens Biograph TOF PET/CT scanners, we consider this effect to be negligible. Lastly, a general limitation of PET/MR imaging is the lack of attenuation correction for coils, which may degrade quantitative assessment of the PET images in clinical studies. Since we are assessing only the PET camera performance in this study and not the MR-based attenuation correction algorithm, we excluded coils from this study. However, this is something to consider in clinical cases when coils are often used. 
PET/MRI scanners from other vendors, such as GE, were not included in this study due to a lack of other vendor sites at the time of conducting this study. Future work will test the performance of GE PET/MRI scanners.

\section{Conclusions}

${ }^{90}$ Y PET measurements from Siemens Biograph mMR (PET/MRI) scanners are acceptable and reproducible at the multi-institutional level. This study may provide insight into the minimum activity concentrations $(\geq 0.60 \mathrm{MBq} / \mathrm{ml})$ and VOI size $(\geq 22 \mathrm{~mm}$ diameter) for accurate and reproducible measurements across institutions. Performance is comparable to that of its TOF PET/CT counterpart and may suggest that multiinstitutional clinical studies of ${ }^{90} \mathrm{Y}$ PET-based dosimetry using Siemens hybrid PET scanners can include both PET/MRI and TOF PET/CT scanners, although MRI may offer additional advantages, such as superior soft-tissue contrast for easy delineation of liver lesions.

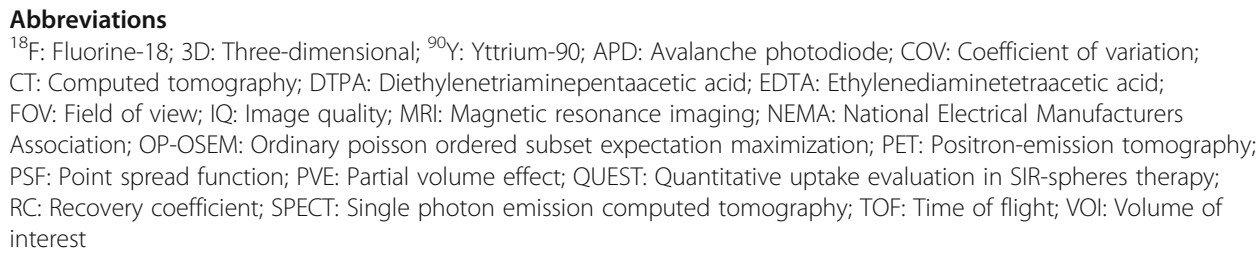

All authors contributed to the data acquisition at their respective institutions. NM, ME, DF, MC, ME, FL, KK, and MT contributed to the design of this study. Data was reconstructed, processed, and analyzed by NM. The manuscript was written by NM. All authors have read and edited the manuscript and consented to its publication.

\section{Ethics approval and consent to participate}

This article does not contain any studies with human participants or animals performed by any of the authors.

\section{Consent for publication}

Not applicable.

\section{Competing interests}

NM has received travel funds from Sirtex. DF, MC, and ML are employees of Siemens Healthineers. MT was a Research and Development manager of Sirtex at the time of data collection; he became an employee of ABX-CRO during manuscript preparation. Though this study was funded by Sirtex Ltd., the study was scientifically conducted independently of said company. The other authors declare that they have no competing interests.

\section{Publisher's Note}

Springer Nature remains neutral with regard to jurisdictional claims in published maps and institutional affiliations.

\section{Author details}

'Department of Radiation Oncology, Washington University School of Medicine, 4921 Parkview Place, Campus Box 8224, St. Louis, MO 63110, USA. ²Translational and Molecular Imaging Institute, Icahn School of Medicine at Mount Sinai, 1470 Madison Ave, New York, NY 10029, USA. ${ }^{3}$ Department of Biomedical Engineering, City College of New York, 160 Convent Ave, New York, NY 10031, USA. ${ }^{4}$ Siemens Healthineers, Siemens Medical Solutions USA, Inc., 40 Liberty Boulevard, Malvern, PA 19355-9998, USA. ${ }^{5}$ Molecular Imaging, Siemens Healthineers, 810 Innovation Dr, Knoxville, TN 37932, USA. ${ }^{6}$ Department of Circulation and Medical Imaging, Faculty of Medicine and Health Sciences, NTNU, Norwegian University of Science and Technology, Postboks 8905, 7491 Trondheim, Norway. ${ }^{7}$ Department of Radiology, Icahn School of Medicine at Mt. Sinai, One G. Levy Pl., Box 1141, New York, NY 10029, USA. ${ }^{8}$ Agency for Science 
Technology and Research, National University of Singapore Clinical Imaging Research Centre, 14 Medical Drive, \#B1-01, Singapore 117599, Singapore. ${ }^{9}$ Department of Nuclear Medicine, Cleveland Clinic, Mail Code Jb3, 9500 Euclid Ave, Cleveland, OH 44195, USA. ${ }^{10}$ NYU Medical Center, 333 E 38th St, New York, NY 10016, USA. ${ }^{11}$ Klinik und Poliklinik für Nuklearmedizin, TU München, Strasse 22, 81675, Munich, Germany. ${ }^{12}$ Department of Nuclear Medicine,

Charité-Universitätsmedizin Berlin, Charitépl. 1, 10117 Berlin, Germany. ${ }^{13}$ Siemens Healthcare GmbH, Berlin, Germany. ${ }^{14}$ Sirtex Medical Ltd, Level 33, 101 Miller St, North Sydney, NSW 2060, Australia. ${ }^{15}$ ABX-CRO Advanced Pharmaceutical Services, 1 Begonia Road, Normanhurst, NSW 2076, Australia. ${ }^{16}$ Mallinckrodt Institute of Radiology, Washington University School of Medicine, 510 S. Kingshighway, Campus Box 8225, St. Louis, MO 63110, USA.

Received: 30 October 2017 Accepted: 16 February 2018

Published online: 04 April 2018

\section{References}

1. Murthy R, Kamat P, Nuñez R, Salem R. Radioembolization of Yttrium-90 microspheres for hepatic malignancy. Semin Interv Radiol. 2008;25(1):48-57. https://doi.org/10.1055/s-2008-1052306.

2. Pasciak AS, Bradley Y, JM MK. Handbook of radioembolization. Physics, Biology, Nuclear Medicine, and Imaging. CRC Press. 2016;

3. Salem R, Thurston KG. Radioembolization with 90 Yttrium microspheres: a state-of-the-art brachytherapy treatment for primary and secondary liver malignancies. Part 1: technical and methodologic considerations. J Vasc Interv Radiol. 2006;17(8):1251-78.

4. van Hazel GA, Heinemann V, Sharma NK, Findlay MPN, Ricke J, Peeters M, et al. SIRFLOX: randomized phase III trial comparing first-line mFOLFOX6 (plus or minus bevacizumab) versus mFOLFOX6 (plus or minus bevacizumab) plus selective internal radiation therapy in patients with metastatic colorectal cancer. J Clin Oncol. 2016;34(15):1723-31. https://doi.org/10.1200/JCO.2015.66.1181.

5. Salem R, Hunter RD. Yttrium-90 microspheres for the treatment of hepatocellular carcinoma: a review. International Journal of Radiation Oncology*Biology*Physics. 2006;66(2, Supplement):S83-S8. https://doi.org/10.1016/j.jirobp.2006.02.061

6. Atassi B, Bangash AK, Bahrani A, Pizzi G, Lewandowski RJ, Ryu RK, et al. Multimodality imaging following $90 \mathrm{Y}$ radioembolization: a comprehensive review and pictorial essay. Radiographics. 2008;28(1):81-99. https://doi.org/10.1148/rg.281065721.

7. Sarfaraz M, Kennedy AS, Lodge MA, Li XA, Wu X, Yu CX. Radiation absorbed dose distribution in a patient treated with yttrium-90 microspheres for hepatocellular carcinoma. Med Phys. 2004;31(9):2449-53. https://doi.org/10.1118/ 1.1781332 .

8. Eaton BR, Kim HS, Schreibmann E, Schuster DM, Galt JR, Barron B et al. Quantitative dosimetry for yttrium-90 radionuclide therapy: tumor dose predicts fluorodeoxyglucose positron emission tomography response in hepatic metastatic melanoma. J Vasc Interv Radiol. 2014;25(2):288-95. https://doi.org/10.1016/j.jvir.2013.08.021.

9. Kokabi N, Galt JR, Xing M, Camacho JC, Barron BJ, Schuster DM et al. A simple method for estimating dose delivered to hepatocellular carcinoma after yttrium-90 glass-based radioembolization therapy: preliminary results of a proof of concept study. J Vasc Interv Radiol. 2014;25(5):277-87. https://doi.org/10.1016/j.jvir.2013.11.007.

10. Kao YH, Steinberg JD, Tay YS, Lim GK, Yan J, Townsend DW, et al. Post-radioembolization yttrium-90 PET/CT-part 1: diagnostic reporting. EJNMMI Res. 2013;3(1):56. https://doi.org/10.1186/2191-219x-3-56.

11. Elschot M, Vermolen BJ, Lam MGEH, de Keizer B, van den Bosch MAAJ, de Jong HWAM. Quantitative comparison of PET and Bremsstrahlung SPECT for imaging the in vivo yttrium-90 microsphere distribution after liver radioembolization. PLoS One. 2013;8(2):e55742. https://doi.org/10.1371/journal.pone.0055742.

12. Lhommel R, van Elmbt L, Goffette P, Van den Eynde M, Jamar F, Pauwels S, et al. Feasibility of 90Y TOF PET-based dosimetry in liver metastasis therapy using SIR-spheres. Eur J Nucl Med Mol Imaging. 2010;37(9):1654-62. https://doi.org/10.1007/s00259-010-1470-9.

13. Gates VL, Esmail AA, Marshall K, Spies S, Salem R. Internal pair production of 90Y permits hepatic localization of microspheres using routine PET: proof of concept. Journal of nuclear medicine : official publication, Society of Nuclear Medicine. 2011;52(1):72-6. https://doi.org/10.2967/jnumed.110.080986.

14. D'Arienzo M, Filippi L, Chiaramida P, Chiacchiararelli L, Cianni R, Salvatori R, et al. Absorbed dose to lesion and clinical outcome after liver radioembolization with $90 \mathrm{Y}$ microspheres: a case report of PET-based dosimetry. Ann Nucl Med. 2013;27(7):676-80. https://doi.org/10.1007/s12149-013-0726-4.

15. Kao YH, Steinberg JD, Tay YS, Lim GK, Yan J, Townsend DW, et al. Post-radioembolization yttrium-90 PET/CT-part 2: dose-response and tumor predictive dosimetry for resin microspheres. EJNMMI Res. 2013;3(1):57. https://doi.org/10.1186/2191-219x-3-57.

16. Fowler KJ, Maughan NM, Laforest R, Saad NE, Sharma A, Olsen J, et al. PET/MRI of hepatic 90Y microsphere deposition determines individual tumor response. Cardiovasc Intervent Radiol. 2016;39(6):855-64 https://doi.org/10.1007/s00270-015-1285-y.

17. van den Hoven AF, Rosenbaum CE, Elias SG, de Jong HW, Koopman M, Verkooijen HM, et al. Insights into the dose-response relationship of radioembolization with resin 90Y-microspheres: a prospective cohort study in patients with colorectal cancer liver metastases. Journal of nuclear medicine : official publication, Society of Nuclear Medicine. 2016;57(7):1014-9. https://doi.org/10.2967/jnumed.115.166942.

18. Willowson KP, Hayes AR, Chan DLH, Tapner M, Bernard EJ, Maher R et al. Clinical and imaging-based prognostic factors in radioembolisation of liver metastases from colorectal cancer: a retrospective exploratory analysis. EJNMMI Res. 2017;7(1):46. https://doi.org/10.1186/s13550-017-0292-1.

19. Fahey FH, Kinahan PE, Doot RK, Kocak M, Thurston H, Poussaint TY. Variability in PET quantitation within a multicenter consortium. Med Phys. 2010;37(7):3660-6. https://doi.org/10.1118/1.3455705.

20. Willowson KP, Tapner M, Bailey DL. A multicentre comparison of quantitative (90)Y PET/CT for dosimetric purposes after radioembolization with resin microspheres : the QUEST phantom study. Eur J Nucl Med Mol Imaging. 2015 42(8):1202-22. https://doi.org/10.1007/s00259-015-3059-9. 
21. Ziegler S, Jakoby BW, Braun H, Paulus DH, Quick HH. NEMA image quality phantom measurements and attenuation correction in integrated PET/MR hybrid imaging. ENMMI Physics. 2015;2(18) https://doi.org/10.1186/s40658-015-0122-3.

22. Maughan NM, Parikh PJ, Laforest R. Low trues statistics and high randoms rates degrade quantitative accuracy in PET iterative reconstruction methods. Nucl Sci Symp-Med Imaging Conference. 2016:M16F-9. http://2016.nss-mic. org/images/program/AbstractBook.pdf.

23. Maughan NM, Eldib M, Conti M, Knešaurek K, Faul D, Parikh PJ, et al. Phantom study to determine optimal PET reconstruction parameters for PET/MR imaging of $90 \mathrm{Y}$ microspheres following radioembolization. Biomedical Physics \& Engineering Express. 2016;2(1):015009.

24. Willowson K, Forwood N, Jakoby BW, Smith AM, Bailey DL. Quantitative (90)Y image reconstruction in PET. Med Phys. 2012;39(11):7153-9. https://doi.org/10.1118/1.4762403.

25. Delso G, Furst S, Jakoby B, Ladebeck R, Ganter C, Nekolla SG, et al. Performance measurements of the Siemens mMR integrated whole-body PET/MR scanner. Journal of nuclear medicine : official publication, Society of Nuclear Medicine. 2011;52(12):1914-22. https://doi.org/10.2967/jnumed.111.092726.

26. Jakoby BW, Bercier Y, Watson CC, Bendriem B, Townsend DW. Performance characteristics of a new LSO PET/CT scanner with extended axial field-of-view and PSF reconstruction. IEEE Trans Nucl Sci. 2009;56(3):633-9. https://doi.org/10.1109/TNS.2009.2015764.

27. Grezes-Besset L, Nuyts J, Boellard R, Buvat I, Michel C, Pierre C et al., editors. Simulation-based evaluation of NEG ML iterative reconstruction of low count PET data. 2007 IEEE nuclear science symposium conference record; 2007 Oct. 26 2007-Nov. 32007.

28. van Velden FHP, Kloet RW, van Berckel BNM, Lammertsma AA, Boellaard R. Accuracy of 3-dimensional reconstruction algorithms for the high-resolution research tomograph. J Nucl Med. 2009;50(1):72-80.

29. Comtat C, Bataille F, Michel C, Jones JP, Sibomana M, Janeiro L et al., editors. OSEM-3D reconstruction strategies for the ECAT HRRT. IEEE Symp Conference Record Nucl Sci 2004. 2004;6:3492-96. https://doi.org/10.1109/NSSMIC. 2004.1466639.

30. Carlier T, Willowson KP, Fourkal E, Bailey DL, Doss M, Conti M. (90)Y -PET imaging: exploring limitations and accuracy under conditions of low counts and high random fraction. Med Phys. 2015;42(7):4295-309. https://doi.org/10.1118/1.4922685.

31. Vandenberghe S, Mikhaylova E, D'Hoe E, Mollet P, Karp JS. Recent developments in time-of-flight PET. EJNMM Physics. 2016;3(1):3. https://doi.org/10.1186/s40658-016-0138-3.

32. Rahmim A, Cheng JC, Blinder S, Camborde ML, Sossi V. Statistical dynamic image reconstruction in state-of-the-art high-resolution PET. 2005(0031-9155 (Print)).

33. Attarwala AA, Molina-Duran F, Büsing K-A, Schönberg SO, Bailey DL, Willowson K, et al. Quantitative and qualitative assessment of yttrium-90 PET/CT imaging. PLoS One. 2014;9(11):e110401. https:/doi.org/10.1371/journal.pone.0110401.

34. Soderlund AT, Chaal J, Tjio G, Totman JJ, Conti M, Townsend DW. Beyond 18F-FDG: characterization of PET/CT and PET/MR scanners for a comprehensive set of positron emitters of growing application-18F, 11C, 89Zr, 124l, 68Ga, and 90Y. J Nucl Med. 2015;56(8):1285-9. https://doi.org/10.2967/jnumed.115.156711.

35. Martinez-Möller A, Zikic D, Botnar RM, Bundschuh RA, Howe W, Ziegler SI, et al. Dual cardiac-respiratory gated PET: implementation and results from a feasibility study. Eur J Nucl Med Mol Imaging. 2007;34(9):1447-54 https://doi.org/10.1007/s00259-007-0374-9.

Submit your manuscript to a SpringerOpen ${ }^{\circ}$ journal and benefit from:

- Convenient online submission

- Rigorous peer review

Open access: articles freely available online

- High visibility within the field

- Retaining the copyright to your article

Submit your next manuscript at $>$ springeropen.com 